\title{
Patient-Reported Lupus Flare Symptoms Are Associated with Worsened Patient Outcomes and Increased Economic Burden
}

\author{
Patricia Katz, PhD; Winnie W. Nelson, PharmD, MS, MBA; R. Paola Daly, MPH; \\ Lauren Topf, BA; Erin Connolly-Strong, PhD; and Michael L. Reed, PhD
}

\begin{abstract}
BACKGROUND: Lupus flares significantly contribute to health resource utilization and hospitalizations. Identification of flare activity may be hindered since validated assessment scales are rarely used in clinical practice and flare severity may fall below clinician-assessed thresholds. Therefore, patient-reported outcomes of lupus flare frequency are important assessment tools for lupus management.

OBJECTIVE: To better understand the relationship between lupus flares as reported by persons with lupus and specific direct and indirect costs, including hospital admission, unplanned urgent care (UC)/emergency department (ED) visits, work productivity loss, and nonwork activity impairment.
\end{abstract}

METHODS: In this cross-sectional analysis, persons with lupus were drawn from 2 enriched sampling sources. Data were collected via an online survey and included individuals with self-reported physician's diagnosis of systemic lupus erythematosus, skin or discoid lupus, or lupus nephritis. Respondents were asked the total number of hospitalizations and ED/UC visits for any reason and for lupus-related hospitalizations and ED/UC visits. Work productivity loss and nonwork activity impairment were measured via the Work Productivity and Activity Impairment-General Health scale. The sample was stratified into those with 0 flares, 1-3 flares, 4-6 flares, and 7 or more flares, with 0 flares used as the reference. Chisquare tests for trend and analyses of variance were used to evaluate differences among flare frequency groups. Multivariable regression modeling was conducted to evaluate the independent relationship of flare frequency to health care use and productivity loss.

RESULTS: We studied 1,288 survey respondents with known flare frequency in the past 12 months. Flare frequency increased with duration of illness. The mean number of lupus-related hospital admissions was significantly associated with increasing flare frequency for the total sample $(F=3.9$; $P<0.009$ ). Compared to patients with no flare, those who reported flare activities had 1.72-3.13 times higher rates of hospitalizations. The mean number of lupus-related ED/UC visits were also found to be significantly associated with increasing flare frequency for the total sample $(F=23.4$; $P<0.001$ ), and rates were increased by 6.98 - to 16.12 -fold for unplanned ED/UC visits depending on flare frequency. Rates of employment were significantly related to increasing flare frequency. With respect to workrelated impairment, absenteeism increased with greater lupus flare frequency $(\mathrm{F}=6.2 ; P<0.001)$, as did presenteeism $(\mathrm{F}=31.5 ; P<0.001)$ and the combined value of total work productivity loss $(\mathrm{F}=30.4 ; P<0.001)$. Mean work-related activity impairment was $12 \%-32 \%$ more among patients who reported flare activities compared to those who reported no flares.

CONCLUSIONS: Increased lupus-related flare frequency is associated with worsened patient outcomes as measured by increased hospitalizations, visits to the ED/UC, work productivity loss, and activity impairment. This association may be an important indicator of disease severity and resource burden and therefore suggests an unmet need among patients experiencing lupus-related flares.

J Manag Care Spec Pharm. 2020;26(3):275-83

Copyright $\odot 2020$, Academy of Managed Care Pharmacy. All rights reserved.

\section{What is already known about this subject}

When measured using conventional scales in clinical trials, lupus flares are associated with increased risk of health care utilization. Current scales to measure flare frequency do not consider interpretation by the patient.

\section{What this study adds}

Flare frequency, as defined by the patient, correlates to increased health care resource use, including increased hospitalization rates, visits to the emergency department and urgent care centers, presenteeism, and absenteeism.

Patient-reported lupus flare frequency may be an indicator of disease severity and resource burden.

S ystemic lupus erythematosus (SLE) disease and flare activity are often assessed in clinical trials using scales such as the Systemic Lupus Erythematosus Disease Activity Index and the British Isles Lupus Assessment Group; however, validated assessments are rarely used in clinical practice to assess flare activity. ${ }^{1}$ An international clinician working group drafted a consensus definition to describe a lupus flare stating, in part, that a flare is a "measurable increase in disease activity and/or laboratory measurements." 2 The working group noted that the lupus flare should be clinically significant by the assessor such that a change in treatment would be deemed necessary.

As evidenced by several international studies, the occurrence of lupus flares significantly contributes to increases in hospitalization rates and health resource utilization. In one retrospective analysis conducted in Spain, severe flares were a significant predictor of increased costs, most of which were attributed to increased hospitalization rates. ${ }^{3}$ In another analysis, lupus-related flares were found to be the most common reason for hospitalization among a large registry of Korean patients. ${ }^{4}$ Likewise, in the ESSENSE study, patients who experienced lupus-related flares had significantly higher rates of unplanned visits to specialists and were more often hospitalized for longer periods of time. ${ }^{5}$ Risk factors for increased hospitalization rates and health care resource utilization for patients experiencing a flare are multifactorial but include missed appointments, elevations in biomarkers of disease activity or severity 
(i.e., serum creatinine, white blood cell counts, and platelets), and immunosuppressive medication use. ${ }^{6}$

While these previous studies have provided evidence of the relationship between flares and health care utilization, the definitions of flare have focused solely on clinically defined flares and have not included the experience of flares from the patient's perspective. Persons with lupus may experience flares in disease activity that fall below clinician-assessed thresholds but may still affect daily function or trigger self-care or health care utilization. The present study seeks to understand the relationship between lupus flares as reported by persons with lupus and specific direct and indirect costs. This crosssectional analysis quantifies the association of patient-reported lupus flare frequency and hospital admissions, unplanned urgent care (UC) and emergency department (ED) visits, and work productivity loss and nonwork activity impairment

\section{Methods}

\section{Ethics}

The study methods, consent form, and survey tool were reviewed by Ethical and Independent Review Services (Independence, $\mathrm{MO}$ ), which granted an exemption from the requirements of federal regulation 45 CFR 46.101(b)(2) and certified the exemption status of the (\#16149-01) on December 20, 2016. Study volunteers read a description of the study and electronically signed an informed consent before initiating the survey.

\section{Study Sample}

This is a cross-sectional study of U.S. adults with lupus drawn from 2 enriched sampling sources. For the first, the patient advocacy group Lupus Foundation of American (LFA) provided an email list of its constituents for outreach to participate. A second source was the nationwide research panel Research Now (Plano, TX), which had accumulated a list of individuals previously screened for a range of comorbid health problems, including lupus. Potential respondents from both sources were contacted by email and invited to participate in the online survey. Respondents were first presented with informed consent and a general health screening question. Those who confirmed a physician diagnosis of systemic lupus erythematosus, skin or discoid lupus, or lupus nephritis continued to the full survey.

To supplement case identification from the LFA cohort, study information was also provided via the LFA newsletter and other social media with a link to the survey. Qualified respondents from the LFA sample who completed the survey were provided with a $\$ 25$ Target gift card. Respondents from the research panel received a redeemable token incentive valued at $\$ 10$, toward a gift of their choice. Demographic characteristics for the lupus population were used as a guide for establishing sampling quotas for gender and age. ${ }^{7}$ Demographic characteristics of respondents were monitored during data collection and sampling fractions were adjusted where possible.
Potential respondents received an initial study invitation and up to 2 reminder invitations.

Completed surveys from both sources were subjected to a series of quality checks to eliminate unreliable study participants. At the beginning and end of the survey, respondents were asked to provide their gender and date of birth, and those providing inconsistent responses were eliminated. The mean time to completion was also used as a quality check, and respondents who finished faster than 1.5 standard deviations (SDs) from the mean time to completion were excluded. The database was further refined by excluding those providing undifferentiated or pattern-based responses (e.g., selecting all listed options or the same option consistently), as well as those who provided incorrect responses to specific requests (e.g., please enter the number 152 in the box below) after receiving online prompts (e.g., please pay closer attention) to maintain the accuracy of their responses.

\section{Variables}

Flares. Information about past year lupus flare frequency and severity was assessed by questions on the number of flares the respondents had experienced and the severity of the most recent flare. Participants were presented with lists of potential flare symptoms and triggers and asked to indicate which were relevant to them. A list of flare management strategies was also presented, and respondents indicated which they used. Potential responses for the list of symptoms and management strategies were developed by consultation with patient groups and clinical researchers and review of existing published literature.

Health Care Resource Use. To assess hospital admissions, respondents were asked about the total number of admissions for any reason in the past 12 months and about the subset of these admissions that were related to the treatment of lupus. Similar survey items were used to obtain total ED and UC visits over the past year and those visits that were related to lupus.

Workplace Productivity. The Work Productivity and Activity Impairment (WPAI) General Health scale was included in the survey to better understand work and activity-related impairment of study respondents. ${ }^{8}$ This instrument includes 6 questions that ascertain the following for the past 7 days: (1) employment status, (2) hours missed due to health problems, (3) hours missed due to other reasons, (4) hours actually worked, (5) degree health affected productivity while working, and (6) degree health was affected during regular (nonwork) activity. Scoring the instrument yields the percentage of time missed due to health (absenteeism), percentage of impairment while working due to health (presenteeism), and overall work impairment due to health (work productivity loss). Percent of nonwork activity impairment due to health is also derived from item 6 alone. 
Patient-Reported Lupus Flare Symptoms Are Associated with Worsened Patient Outcomes and Increased Economic Burden

TABLE 1 Demographics and Disease Characteristics for Respondents with Known Flare Frequency During the Past Year $(\mathrm{N}=1,288)$

\begin{tabular}{|c|c|c|c|c|c|c|c|c|c|c|c|c|}
\hline \multirow[b]{2}{*}{ Women, n (\%) } & \multicolumn{2}{|c|}{$\begin{array}{c}\text { Total } \\
(\mathrm{N}=1,288)\end{array}$} & \multicolumn{2}{|c|}{$\begin{array}{c}\text { No Flares } \\
(\mathrm{n}=189)\end{array}$} & \multicolumn{2}{|c|}{$\begin{array}{c}\text { 1-3 Flares } \\
(\mathrm{n}=563)\end{array}$} & \multicolumn{2}{|c|}{$\begin{array}{c}\text { 4-6 Flares } \\
(\mathrm{n}=291)\end{array}$} & \multicolumn{2}{|c|}{$\begin{array}{c}\geq 7 \text { Flares } \\
(\mathrm{n}=245)\end{array}$} & \multirow{2}{*}{$\frac{\chi^{2 / F}}{19.7}$} & \multirow{2}{*}{$\frac{P \text { Value }}{<0.001}$} \\
\hline & 1,162 & $(90.2)$ & 162 & $(85.7)$ & 495 & (87.9) & 269 & (92.4) & 236 & (96.3) & & \\
\hline Age, mean (SD) & 45.2 & (13.3) & 46.3 & (14.8) & 44.8 & (13.3) & 45.8 & (13.1) & 44.4 & $(12.2)$ & $\mathrm{F}=1.025$ & $<0.001$ \\
\hline \multicolumn{13}{|l|}{ Race, n (\%) } \\
\hline White & 1,001 & $(82.0)$ & 154 & $(85.6)$ & 423 & $(79.4)$ & 227 & $(81.7)$ & 197 & $(86.0)$ & \multirow{3}{*}{10.831} & \multirow{3}{*}{0.094} \\
\hline African American & 173 & $(14.2)$ & 17 & (9.4) & 92 & (17.3) & 40 & $(14.4)$ & 24 & $(10.5)$ & & \\
\hline Other & 46 & (3.8) & 9 & $(5.0)$ & 18 & (3.4) & 11 & $(4.0)$ & 8 & $(3.5)$ & & \\
\hline Hispanic ethnicity, n (\%) & 156 & $(12.2)$ & 30 & $(16.0)$ & 62 & $(11.0)$ & 34 & $(11.8)$ & 30 & $(12.3)$ & 3.244 & 0.356 \\
\hline Married, n (\%) & 743 & $(58.4)$ & 122 & $(65.2)$ & 326 & $(58.5)$ & 174 & $(60.2)$ & 121 & $(50.4)$ & 10.287 & 0.016 \\
\hline \multicolumn{13}{|l|}{ Household income, $\mathrm{n}(\%)$} \\
\hline$<\$ 25,000$ & 263 & $(22.3)$ & 20 & (11.2) & 96 & (18.6) & 72 & $(27.7)$ & 75 & $(33.5)$ & \multirow{5}{*}{72.856} & \multirow{5}{*}{$<0.001$} \\
\hline$\$ 25,000-\$ 49,999$ & 294 & (25) & 39 & (21.8) & 141 & (27.4) & 54 & (20.8) & 60 & (26.8) & & \\
\hline$\$ 50,000-\$ 74,999$ & 199 & (16.9) & 40 & $(22.3)$ & 76 & (14.8) & 37 & (14.2) & 46 & $(20.5)$ & & \\
\hline$\$ 75,000-\$ 99,000$ & 148 & $(12.6)$ & 19 & $(10.6)$ & 71 & (13.8) & 43 & $(16.5)$ & 15 & $(6.7)$ & & \\
\hline$\geq \$ 100,000$ & 274 & $(23.3)$ & 61 & $(34.1)$ & 131 & $(25.4)$ & 54 & $(20.8)$ & 28 & $(12.5)$ & & \\
\hline \multicolumn{13}{|l|}{ Census region, $\mathbf{n}(\%)$} \\
\hline Northeast & 211 & (16.4) & 35 & $(18.5)$ & 96 & $(17.1)$ & 42 & (14.4) & 38 & (15.5) & \multirow{4}{*}{19.527} & \multirow{4}{*}{0.021} \\
\hline Midwest & 286 & $(22.2)$ & 48 & $(25.4)$ & 136 & $(24.2)$ & 57 & $(19.6)$ & 45 & $(18.4)$ & & \\
\hline South & 487 & $(37.8)$ & 56 & (29.6) & 214 & $(38.0)$ & 107 & (36.8) & 110 & $(44.9$ & & \\
\hline West & 304 & $(23.6)$ & 50 & $(26.5)$ & 117 & (20.8) & 85 & $(29.2)$ & 52 & $(21.2)$ & & \\
\hline Employment status ${ }^{\mathrm{a}}, \mathrm{n}(\%)$ & 636 & $(49.4)$ & 120 & $(63.5)$ & 304 & $(54.0)$ & 124 & $(42.6)$ & 88 & $(35.9)$ & 42.953 & $<0.001$ \\
\hline Working for pay (full or part) & 610 & $(47.4)$ & 115 & $(60.8)$ & 292 & $(51.9)$ & 120 & $(41.2)$ & 83 & (33.9) & 3.132 & $<0.001$ \\
\hline Disabled: Working for pay & 35 & $(2.7)$ & 8 & $(4.2)$ & 14 & $(2.5)$ & 5 & $(1.7)$ & 8 & (3.3) & 58.193 & 0.372 \\
\hline Disabled: Not working & 329 & $(25.5)$ & 12 & $(6.3)$ & 135 & $(24.0)$ & 94 & $(32.3)$ & 88 & $(35.9)$ & 3.704 & $<0.001$ \\
\hline Only temporarily laid off & 8 & $(0.6)$ & 3 & (1.6) & 2 & $(0.4)$ & 2 & $(0.7)$ & 1 & $(0.4)$ & 6.511 & 0.295 \\
\hline On sick or maternity leave & 24 & (1.9) & 2 & $(1.1)$ & 6 & $(1.1)$ & 8 & $(2.7)$ & 8 & (3.3) & 2.016 & 0.089 \\
\hline Looking for work/unemployed & 59 & $(4.6)$ & 7 & $(3.7)$ & 23 & $(4.1)$ & 14 & $(4.8)$ & 15 & $(6.1)$ & 8.209 & 0.569 \\
\hline Retired & 149 & $(11.6)$ & 33 & $(17.5)$ & 59 & $(10.5)$ & 34 & $(11.7)$ & 23 & $(9.4)$ & 8.943 & 0.042 \\
\hline Homemaker & 124 & $(9.6)$ & 11 & $(5.8)$ & 47 & $(8.3)$ & 34 & (11.7) & 32 & $(13.1)$ & 4.416 & 0.030 \\
\hline Student & 58 & $(4.5)$ & 8 & $(4.2)$ & 31 & $(5.5)$ & 7 & $(2.4)$ & 12 & $(4.9)$ & 4.052 & 0.220 \\
\hline BMI, mean (SD) & 29.5 & $(7.9)$ & 27.5 & $(6.2)$ & 29.6 & $(8.0)$ & 29.6 & $(7.7)$ & 30.8 & $(8.7)$ & $F=6.489$ & $<0.001$ \\
\hline Current smoker, n (\%) & 127 & $(9.9)$ & 7 & $(3.7)$ & 46 & $(8.2)$ & 34 & $(11.7)$ & 40 & $(16.3)$ & 22.483 & $<0.001$ \\
\hline Duration of illness, mean years (SD) & 11.9 & (9.8) & 15.9 & $(11.2)$ & 12.2 & $(10.1)$ & 11.1 & $(0.5)$ & 9.0 & $(8.1)$ & $\mathrm{F}=18.522$ & $<0.001$ \\
\hline Medication use, n (\%) & & & & & & & & & & & & \\
\hline Steroid & 540 & $(41.9)$ & 46 & $(24.3)$ & 258 & $(45.8)$ & 123 & $(42.3)$ & 113 & $(46.1)$ & 29.313 & $<0.001$ \\
\hline Biologic & 199 & (15.5) & 18 & $(9.5)$ & 95 & (16.9) & 45 & $(15.5)$ & 41 & $(16.7)$ & 6.265 & 0.099 \\
\hline DMARD & 885 & $(68.7)$ & 98 & $(51.9)$ & 389 & $(69.1)$ & 205 & $(70.4)$ & 193 & (78.8) & 36.977 & $<0.001$ \\
\hline GABA analog & 204 & (15.8) & 7 & $(3.7)$ & 93 & $(16.5)$ & 60 & $(20.6)$ & 44 & (18.0) & 26.889 & $<0.001$ \\
\hline Immunosuppressant & 197 & (15.3) & 28 & (14.8) & 99 & $(17.6)$ & 37 & $(12.7)$ & 33 & $(13.5)$ & 4.437 & 0.218 \\
\hline Hormone & 55 & $(4.3)$ & 4 & $(2.1)$ & 24 & $(4.3)$ & 15 & $(5.2)$ & 12 & $(4.9)$ & 2.938 & 0.401 \\
\hline Other lupus drug & 81 & (6.3) & 12 & (6.3) & 40 & (7.1) & 21 & (7.2) & 8 & (3.3) & 4.863 & 0.182 \\
\hline Number of lupus drugs $\mathrm{u}$ & & & & & & & & & & & & \\
\hline No lupus drug use & 166 & (12.9) & 46 & $(24.3)$ & 56 & (9.9) & 32 & (11) & 32 & $(13.1)$ & & \\
\hline Using 1 lupus drug & 474 & (36.8) & 90 & $(47.6)$ & 206 & (36.6) & 106 & (36.4) & 72 & (29.4) & 56.098 & $<0.001$ \\
\hline Using 2 or more lupus drugs & 648 & $(50.3)$ & 53 & (28) & 301 & $(53.5)$ & 153 & $(52.6)$ & 141 & $(57.6)$ & & \\
\hline Severity of most recent flare, $n(\%)$ & & & & & & & & & & & & \\
\hline Mild & 95 & $(8.8)$ & & & 72 & $(13.1)$ & 17 & $(5.9)$ & 6 & $(2.5)$ & & \\
\hline Mild to moderate & 257 & $(23.7)$ & & & 177 & $(32.2)$ & 56 & (19.3) & 24 & $(9.8)$ & & \\
\hline Moderate & 332 & $(30.7)$ & & & 163 & $(29.6)$ & 98 & (33.8) & 71 & $(29.1)$ & 126.131 & $<0.001$ \\
\hline Moderate to severe & 291 & $(26.8)$ & & & 98 & $(17.8)$ & 96 & $(33.1)$ & 97 & $(39.8)$ & & \\
\hline Severe & 109 & (10.1) & & A & 40 & (7.3) & 23 & (7.9) & 46 & (18.9) & & \\
\hline
\end{tabular}

aWorking for pay (full or part time) and disabled (working for pay) combined.

$B M I=$ body mass index; $D M A R D=$ disease-modifying antirheumatic drug; $G A B A=$ gamma-aminobutyric acid; $N A=$ not applicable; $S D=$ standard deviation;

SLE = systemic lupus erythematous. 
Patient-Reported Lupus Flare Symptoms Are Associated with Worsened Patient Outcomes and Increased Economic Burden

TABLE 2 Lupus-Related Hospitalizations, Lupus-Related ED/UC Visits, and Activity Impairment for the Total Sample with Known Flare Frequency $(\mathrm{N}=1,288)$ and Absenteeism, Presenteeism, Total Work Productivity Loss, and Activity Impairment for the Employed Sample ( $\mathrm{N}=636$ )

\begin{tabular}{|c|c|c|c|c|c|c|c|}
\hline $\begin{array}{l}\text { Total Sample with } \\
\text { Known Flare Frequency }\end{array}$ & $\begin{array}{c}\text { Total } \\
(\mathrm{N}=1,288)\end{array}$ & $\begin{array}{l}\text { No Flares } \\
(\mathrm{n}=189)\end{array}$ & $\begin{array}{l}1-3 \text { Flares } \\
(\mathrm{n}=563)\end{array}$ & $\begin{array}{l}\text { 4-6 Flares } \\
(\mathrm{n}=291)\end{array}$ & $\begin{array}{l}\geq 7 \text { Flares } \\
(\mathrm{n}=245)\end{array}$ & $\begin{array}{l}\text { ANOVA } \\
\text { F-Test }\end{array}$ & $P$ Value \\
\hline $\begin{array}{l}\text { Lupus-related hospital admissions in } \\
\text { the past } 12 \text { months, mean (SD) }\end{array}$ & $0.38 \quad(0.98)$ & $0.22 \quad(0.71)$ & $0.36 \quad(0.75)$ & $0.39 \quad(1.11)$ & $0.53 \quad(1.38)$ & 3.855 & 0.009 \\
\hline $\begin{array}{l}\text { Lupus-related ED/UC visits in the } \\
\text { past } 12 \text { months, mean (SD) }\end{array}$ & $0.95 \quad(2.86)$ & $0.09 \quad(0.75)$ & $0.72 \quad(2.13)$ & $0.91 \quad(2.52)$ & $2.18 \quad(4.74)$ & 23.355 & $<0.001$ \\
\hline $\begin{array}{l}\text { WPAI percent activity impairment } \\
\text { (nonwork), mean (SD) }\end{array}$ & $51.58(27.34)$ & $29.74(27.04)$ & $48.33(26.57)$ & $58.80(22.18)$ & $67.31(21.58)$ & 93.048 & $<0.001$ \\
\hline Employed Sample $^{a}$ & $\begin{array}{c}\text { Total } \\
(\mathrm{N}=636)\end{array}$ & $\begin{array}{l}\text { No Flares } \\
(\mathrm{n}=120)\end{array}$ & $\begin{array}{l}\text { 1-3 Flares } \\
(\mathrm{n}=304)\end{array}$ & $\begin{array}{c}\text { 4-6 Flares } \\
(\mathrm{n}=124)\end{array}$ & $\begin{array}{c}\geq 7 \text { Flares } \\
\quad(n=88)\end{array}$ & $\begin{array}{c}\text { ANOVA } \\
\text { F-Test }\end{array}$ & $P$ Value \\
\hline $\begin{array}{l}\text { Percent of time missed due to health } \\
\text { (absenteeism) }\end{array}$ & $11.27(20.04)$ & $6.79(15.76)$ & $9.94(19.66)$ & $14.32(21.00)$ & $17.52(23.11)$ & 6.207 & $<0.001$ \\
\hline $\begin{array}{l}\text { Percent of time impaired while working } \\
\text { due to health (presenteeism) }\end{array}$ & $37.98(25.43)$ & $24.17(25.48)$ & $35.21(24.36)$ & $47.69(22.39)$ & $52.53(20.41)$ & 31.515 & $<0.001$ \\
\hline $\begin{array}{l}\text { Percent overall work impairment due to } \\
\text { health (work productivity loss) }\end{array}$ & $43.11(28.79)$ & $27.82(28.97)$ & $40.04(28.18)$ & $53.48(24.17)$ & $59.57(23.04)$ & 30.361 & $<0.001$ \\
\hline Percent activity impairment (non-work) & $43.87(27.12)$ & $28.83(26.70)$ & $40.66(26.56)$ & $53.95(22.84)$ & $61.25(20.05)$ & 36.797 & $<0.001$ \\
\hline
\end{tabular}

aWorking for pay (full or part time) and disabled (working for pay) combined.

ANOVA = analysis of variance; ED=emergency department; SD=standard deviation; UC= urgent care; WPAI= Work Productivity and Activity Impairment

Other Variables. In addition to the primary areas of focus, the survey captured data on a range of domains including sociodemographic characteristics, perceptions of disease severity, quality of life, pain interference, comorbid health problems, fatigue, psychological symptoms, disease-related burden, family role functioning, flare frequency and severity, and medication use and adherence. Sociodemographic information included age, sex, race and ethnicity, marital status, household income, census region, and employment status. Current tobacco use was assessed by asking respondents if they smoked (yes or no) at the time the survey was administered. Body mass index (BMI) was calculated in the usual manner by dividing weight in pounds by height in inches squared and multiplying by a conversion factor of 703 . The survey assessed the respondents' duration of illness and current medication use (within the past 3 months to manage lupus). A list of medications (Appendix, available in online article) was included as part of the survey to minimize recall bias. Medications were grouped as follows for the analysis: steroid and corticosteroid medications, biologics, other disease-modifying antirheumatic drugs (DMARDs), gamma-aminobutyric acid (GABA) analogs, immunosuppressants, and hormones.

\section{Statistical Analysis}

Analyses were performed using IBM SPSS Statistics Version 24.0.0 (IBM, Armonk, NY). The aim of this analysis was to better understand the relationship between the frequency and pattern of lupus symptom flares from the perception of people with lupus and to assess the association of flare frequency on hospital admissions, unplannedED/UC visits, and work productivity loss and nonwork activity impairment. The sample was stratified into those with 0 flares, 1-3 flares, 4-6 flares, and 7 or more flares among the subset of respondents confirming recall of lupus flare activity over the previous 12 months. Descriptive statistics were generated for all respondents and for each flare frequency group. Percentages were reported for flare frequency, severity and symptom characteristics, medication use, any hospitalization, any ED/UC visit, and for all sociodemographic characteristics, except for age and BMI, where mean (SD) values were provided. Mean and SD values were also calculated for past year hospital admissions, past year ED/UC visits, and for the work productivity loss and nonwork activity impairment scores from the WPAI. Chi-square test for trend and analysis of variance were used to evaluate differences among flare frequency groups. Since the primary focus was the pattern of findings, post-hoc testing was not implemented.

Regression modeling was used to more fully understand the relationship between flare frequency and the outcomes variables. Model selection was based on the sample distributions for the variables of interest. A 2-stage approach was used. In an initial model, the following variables were included in addition to flare frequency: age, sex, race (African American, other vs. Caucasian), marital status (married vs. not), SLE versus other type of lupus, household income, smoking, duration of illness (years), and BMI. Noncontributing variables $(P<0.05)$ were eliminated, and a second trimmed model included the remaining variables and compared flare frequency groups using the zero recent flare group ( 0 flares in past 12 months) as the reference. For hospital admissions and for ED/UC visits, the large number of zero values required a negative binomial generalized 
Patient-Reported Lupus Flare Symptoms Are Associated with Worsened Patient Outcomes and Increased Economic Burden

FIGURE 1 Adjusted Rate Ratio (95\% Cl) of Lupus-Related Hospital Admissions in the Past 12 Months (N=1,288)

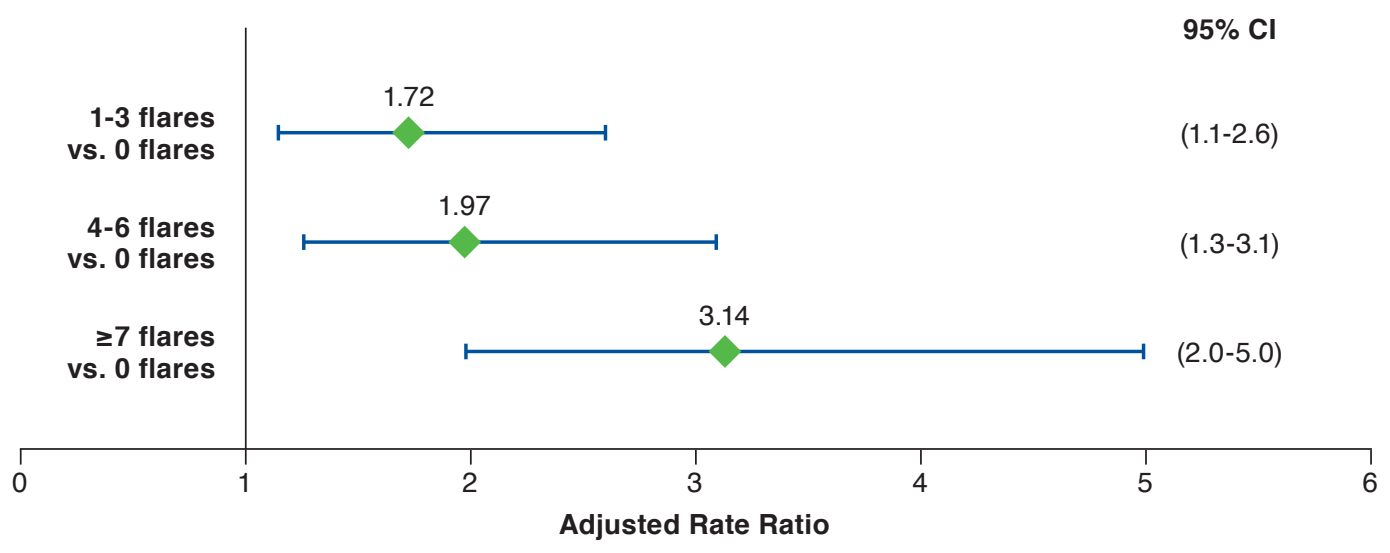

Note: $P<0.05$ relative to no flares for all comparisons. Model was adjusted for gender, race, age, household income, and BMI $B M I=$ body mass index; $C I=$ confidence interval.

linear model with a log link. The distributions for work productivity loss data were normally distributed and linear regression modeling was used. The missing data rate for any single variable was $5 \%$ or lower and imputation was not required. ${ }^{9}$

\section{Results}

\section{Sample Characteristics}

Completed surveys totaled 1,012 from LFA members or affiliates and 595 from the research panel. Due to the varied recruiting methods and our inability to confirm who received an email invitation to participate, it was not possible to estimate a survey response rate. Quality control and internal consistency checks were implemented as well as a check for duplicate respondents. No duplicate respondents were identified, and a total of 939 LFA (92.8\%) and 564 (94.8\%) research panel respondents were retained after quality checks. This yielded a sample of 1,503, of which 1,288 (85.7\%) had known flare frequency in the past 12 months and provided data for the analysis of hospital admissions and ED/UC visits. Data from a further subset of 636 (49.4\%) employed respondents were used for the analysis of work-related productivity loss. The assessment of nonwork-related activity loss was evaluated for the total sample. The survey was fielded between May and October 2017, with an average completion time of 35 minutes. Mean age of the 1,288 total sample with known flare frequency was 45.2 years, $90.2 \%(1,162)$ were women, $82.0 \%(1,001)$ were white, and $58.4 \%$ (743) were married (Table 1).

\section{Factors Associated with Flare Frequency}

Women were more likely to present in the higher-frequency flare groups, but race, Hispanic ethnicity, and marital status did not differ significantly across flare groups (Table 1). Mean age varied significantly among flare groups but the trend was not directional. As flare frequency increased, there was a trend toward lower incomes and lower rates of employment. Rates of smoking increased as flare frequency increased, as did mean BMI.

Duration of lupus was inversely related to flare frequency. Higher rates of steroid, DMARD, and GABA analog use were noted among those who reported flares, as were higher rates of using multiple lupus medications. Increasing flare frequency was also associated with reports of more severe flare activity.

\section{Hospital Admissions}

The mean number of lupus-related hospital admissions was significantly associated with increasing flare frequency (Table 2). An initial multivariable regression model looking at the relationship between flare frequency and the number of hospital admissions identified nonsignificant $(P<0.05)$ covariates (marital status, smoking status, diagnosis, duration of illness), which were trimmed. With the remaining covariates in the model (gender, race, age, household income, and BMI), respondents who reported 1-3, 4-6, and $\geq 7$ flares in the past 12 months had adjusted rate ratio (95\% confidence interval [CI]) increases of 1.72-fold (1.1-2.6), 1.97-fold (1.2-3.1), and 3.14-fold (2.0-5.0), respectively, in the number of hospital admissions visits relative to those with no flares (Figure 1). It should be noted that due to the large number of zero hospital admissions, the flare group means (SDs) are small, ranging from $0.22(0.71)$ for the zero flares past year group to 0.53 (1.38) for the $\geq 7$ flares group.

\section{ED/UC Visits}

The mean number of lupus-related ED/UC visits was also found to be significantly associated with increasing flare frequency for the total sample with known flare frequency (Table 2). 
Patient-Reported Lupus Flare Symptoms Are Associated with Worsened Patient Outcomes and Increased Economic Burden

FIGURE 2 Adjusted Rate Ratio (95\% Cl) of Lupus-Related ED/UC Visits in the Past 12 Months ( $\mathrm{n}=1,288)$

$95 \% \mathrm{Cl}$

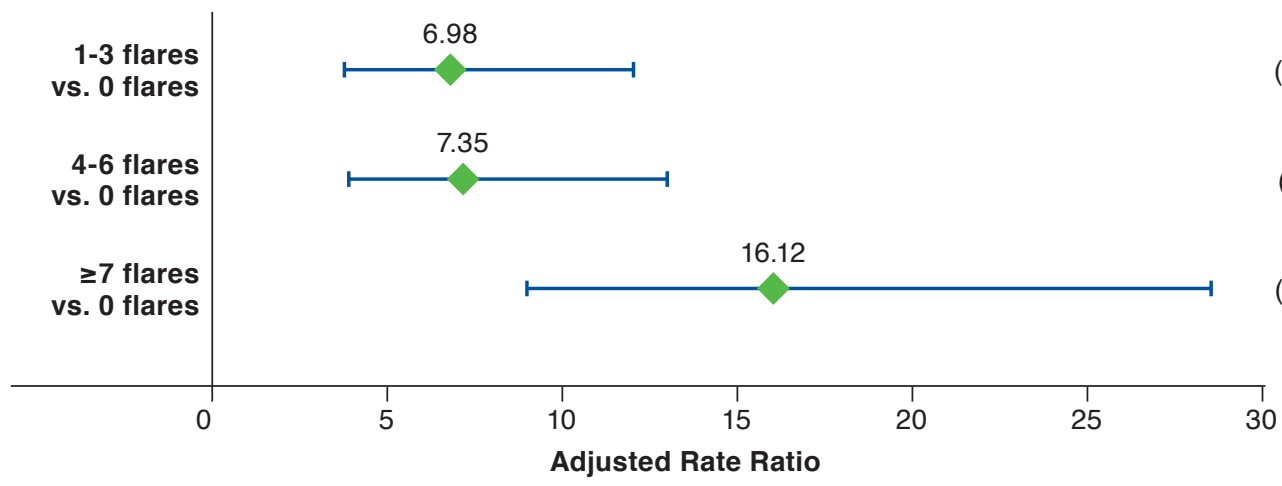

$(4.0-12.2)$

$(4.1-13.1)$

$(9.1-28.6)$ $C I=$ confidence interval; $E D=$ emergency department; $U C=$ urgent care.

A regression model of these data assessed the relationship between flare frequency and unplanned visits to the ED/UC. As before, the nonsignificant $(P<0.05)$ covariates (smoking status, BMI) were identified in an initial model and removed. With the remaining covariates in the model (gender, race, marital status, diagnosis, age, household income, and duration of illness), respondents who reported flares were 7 to 16 times more likely to have unplanned ED/UC visits (adjusted rate ratio [95\% CI] for 1-3 flares: 6.98 [4.0-12.2], $P<0.00$; 4-6 flares: 7.35 [4.1-13.1], $P<0.001$; $\geq 7$ flares: 16.12 [9.1-28.6] $P<0.001$; Figure 2).

\section{Work Productivity Loss and Nonwork Activity Impairment}

For the total sample, we evaluated percent of time respondents reported nonwork activity impairment from the WPAI and found greater nonwork activity impairment with increasing flare frequency (Table 2).

Examining the employed subsample alone ( $n=636)$, absenteeism increased with greater lupus flare frequency, as did presenteeism and the combined value of total work productivity loss (Table 2). A multivariable regression model assessed the relationship between flare frequency and total work productivity loss in the past week. As in the previous models, the nonsignificant $(P<0.05)$ covariates (race, marital status, smoking status, diagnosis, duration of illness, BMI, and household income) were identified in a preliminary model and removed. With the remaining covariates in the model (gender and age), respondents who reported 0 flares had on average $28.7 \%$ work productivity loss, those reporting 1-3 flares had on average $41.4 \%$ work productivity loss, respondents reporting 4-6 flares had on average $54.8 \%$ work productivity loss, and respondents reporting $\geq 7$ flares in the past 12 months had on average
$61.9 \%$ work productivity loss (Figure 3). The relationship between flare frequency and nonwork impairment among the employed group was similar to that noted in the total sample.

\section{Discussion}

In this large cross-sectional analysis of adults with lupus, the associations were quantified between the frequency of patientreported lupus flares and the number of hospitalizations, number of unplanned ED/UC visits, work productivity loss, and nonwork activity impairment. Among the 1,288 respondents with known flare frequency in the past year, the mean number of lupus-related hospital admissions and ED/UC visits incrementally and significantly increased as flare frequency increased. Compared to patients with no flare, those who reported flares had 1.72-3.14 times higher rates of hospitalizations and 6.98-16.12 times higher rates of unplanned ED/UC visits. In addition, work productivity loss was 13\%-33\% greater among patients who reported flare activity compared with those who reported no flares.

These study findings indicate that patient-reported lupus flare may serve as a risk marker for health care resource use and work productivity loss. Hospitalizations have been reported to occur in 22\%-28\% of lupus patients annually. ${ }^{10}$ Lupus was also ranked as the sixth highest readmission rate among all medical conditions in the United States based on 2010 Healthcare Cost and Utilization Project data. ${ }^{11}$ Data here suggest that patient-reported flares, whether accompanied by clinical assessments or not, may be important predictors of high health care resource use, particularly among individuals with a high number of flares.

Lupus has a considerable impact on work productivity, specifically in the employed population. Studies suggest that 
within 5 years of diagnosis, $15 \%-40 \%$ of patients with lupus are unemployed. ${ }^{12-14}$ Further, lupus contributes to a mean annual productivity cost of $\$ 8,659$, with higher costs associated with greater disease activity and worse health. ${ }^{15}$ While there is no published literature on the relationship between lupus-related flare activity and work productivity, previous research on SLE suggests that resulting long-term morbidity subsequently contributes to loss of work productivity. ${ }^{14,16,17}$ To our knowledge, this is the first analysis conducted to correlate flare frequency in lupus with workplace burdens. Data here indicate that among the employed sample $(n=636)$, nonwork activity impairment and work-related absenteeism, presenteeism, and work productivity loss were significantly affected by flare frequency. For work productivity loss, relative to no flares, those reporting 1-3 flares had a $12.6 \%$ more work productivity loss. This increased to $33.1 \%$ for those reporting more than 7 flares.

The sociodemographic characteristics of this analysis such as gender (approximately 90\% women) and age (mean age $=45$ years) appeared to be representative of the lupus population, although individuals of racial/ethnic minorities may have been under-represented (the sample was $77 \%$ white) ${ }^{18}$ Our univariate analyses reported several significant correlations between respondent characteristics and flare frequency. Particularly of interest are modifiable factors including BMI and smoking. Our study observed that increases in BMI were associated with increases in flare frequency. This correlation aligns with a previous report from a Canadian cohort suggesting that BMI is a risk factor for hospitalizations associated with lupus flare. ${ }^{19}$ Smoking is a substantial public health challenge, and it may contribute to cumulative damage to lupus patients. In a cross-sectional study of 105 lupus patients in Brazil, patients who were never exposed to smoking had a 22\% lower risk of chronic cumulative damage, as measured by the Systemic Lupus International Collaborating Clinics/American College of Rheumatology Damage Index score. ${ }^{20}$ Our study findings provide further evidence that weight management and smoking cessation may contribute to better disease management in lupus patients.

In a Medicaid database analysis of 14,777 patients with SLE, $97 \%$ of patients experienced at least 1 flare during the 38.8month follow-up period. ${ }^{21}$ Flares ranged in severity based on drug regimen responses (e.g., initiation of hydroxychloroquine or low-dose steroid was considered as a mild flare and highdose prednisone was categorized as a severe flare), and since more than 1 flare could occur during the study period, patients reported mild (75\%), moderate (91\%), or severe (23\%) flares. Health care cost was directly related to the severity of flares. The cost per episode was $\$ 11,716$ for severe flares, $\$ 562$ for moderate flares, and $\$ 129$ for mild flares. Furthermore, annual total medical costs, which included SLE- and non-SLE-related costs, were significantly greater for those who experienced severe flares $(\$ 49,754)$ relative to those who experienced
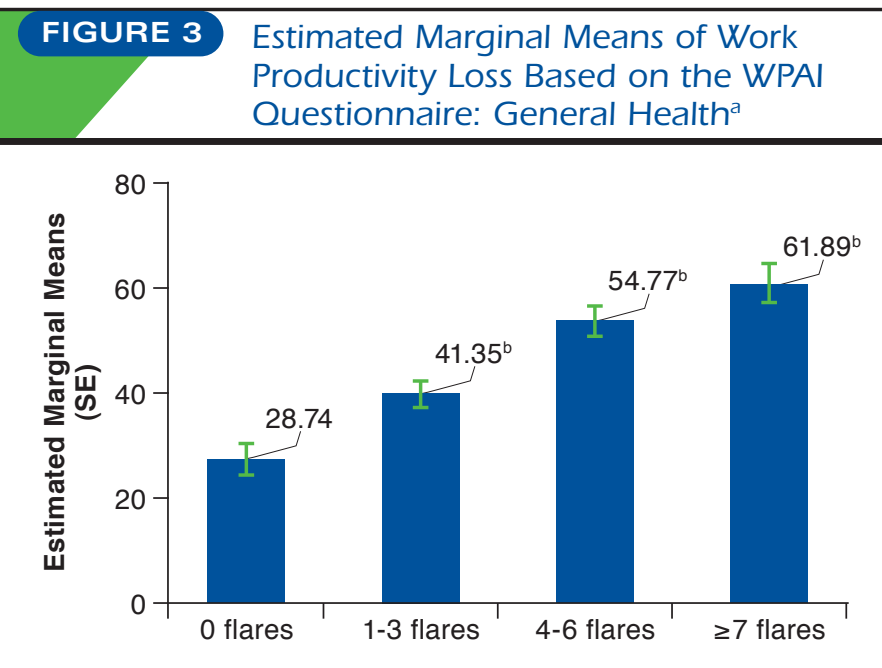

${ }^{a}$ Error bars represent standard error for employed respondents $(n=636)$. ${ }^{b} \mathrm{P}<0.001$ for all flare groups relative to no flares.

SE $=$ standard error; WPAI = Work Productivity and Activity Impairment.

moderate $(\$ 21,941)$ and mild $(\$ 17,574)$ flares. Interestingly, the number of patients reporting more than 3 flares per year was less in the severe flare group (12\%) relative to the mild (55\%) or moderate flare $(75 \%)$ groups, suggesting that the number of flares did not necessarily correlate with flare intensity, although a lower frequency in the higher severity group may have been balanced by longer time to flare resolution. In contrast, our analysis found baseline flare frequency was associated with significantly more severe flare activity. Nonetheless, flare intensity in the analysis by Kan et al. (2013) and flare frequency (as reported in this analysis) both appear to increase total health care costs. ${ }^{21}$

\section{Limitations}

There are several limitations to this analysis that should be noted. Most obviously, diagnosis was self-reported; however, respondents confirmed diagnostic status from a list of precoded conditions. In addition, the number of reported flares was determined by the patient and is limited by patient recall bias. It should also be noted that our measure of productivity loss is limited to the past 7 days, while flare frequency is measured over the past 12 months, and productivity may be affected by other factors and comorbid health problems that we did not capture. Despite these potential limitations, findings in this study showed the value of patient-reported lupus flare, giving credence to the need to incorporate such measures in clinical care.

Population-based surveys, such as those used in this analysis, may not capture the most severe cases of lupus because patients with severe disease may find it difficult to participate because of their illness or they may be hospitalized. In addition 
to clinical factors that may foreshadow flares, such as serologic measures, other factors associated with risk of flares, such as pre-flare adherence to medications, may not have been comprehensively assessed. Furthermore, certain populations such as those who may be in the military, non-English speaking, some racial/ethnic groups, or from lower socioeconomic status classes were under-represented in this analysis. Further analysis of specific populations may be warranted.

\section{Conclusions}

Increased lupus-related flare frequency is associated with worsened patient outcomes as measured by increased hospitalizations, visits to the ED/UC, and work productivity loss. A patient-reported lupus flare is an observable event and may be an important indicator of disease severity and resource burden. Timely response with access to treatment options and implementation of preventive strategies may help reduce health care resource use and work productivity loss.

Authors

PATRICIA KATZ, PhD, University of California, San Francisco WINNIE W. NELSON, PharmD, MS, MBA, and ERIN CONNOLLYSTRONG, PhD, Mallinckrodt Pharmaceuticals, Bedminster, New Jersey. R. PAOLA DALY, MPH, and LAUREN TOPF, BA, The Lupus Foundation of America, Washington, DC. MICHAEL L. REED, PhD, Vedanta Research, Chapel Hill, North Carolina.

AUTHOR CORRESPONDENCE: Patricia Katz, PhD, University of California San Francisco, Arthritis Research Group, 3333 California St., San Francisco, CA 94143-0936. Tel.: 415.476.5971;

E-mail:Patti.Katz@ucsf.edu.

\section{DISCLOSURES}

This study was sponsored by Mallinckrodt Pharmaceuticals via grants to Vedanta Research and The Lupus Foundation of America. Katz received consulting fees from Vedanta Research, which received grant support from Mallinckrodt Pharmaceuticals to support data collection and analysis. Nelson and Connolly-Strong are employees of Mallinckrodt Pharmaceuticals and are stockholders in the company. Reed is an employee of Vedanta Research. Daly and Topf are employees of the Lupus Foundation of America, which received grant funding to support data collection.

This study was a podium presentation at The American College of Rheumatology (ACR) Annual Meeting 2018; October 19-24, 2018; Chicago, IL.

\section{ACKNOWLEDGMENTS}

Valerie Marske of Vedanta Research supported survey instrument development and data collection; Kristina Fanning, PhD, and Ryan Bostic of Vedanta Research provided analytical support; and Dana A. Franznick, PharmD, and Ishveen Chopra provided medical writing support.

\section{REFERENCES}

1. Isenberg D, Sturgess J, Allen E, et al. Study of flare assessment in systemic lupus erythematosus based on paper patients. Arthritis Care Res. 2018; 70(1):98-103.

2. Ruperto N, Hanrahan LM, Alarcon GS, et al. International consensus for a definition of disease flare in lupus. Lupus. 2011;20(5):453-62.

3. Cervera R, Rua-Figueroa I, Gil-Aguado A, et al. Direct cost of management and treatment of active systemic lupus erythematosus and its flares in Spain: the LUCIE study. Revista Clinica Espanola. 2013;213(3):127-37.

4. Lee JW, Park DJ, Kang JH, et al. The rate of and risk factors for frequent hospitalization in systemic lupus erythematosus: results from the Korean lupus network registry. Lupus. 2016;25(13):1412-19.

5. Nasonov E, Soloviev S, Davidson JE, et al. Systemic lupus erythematosus and associated healthcare resource consumption in selected cities from the Russian Federation, Republic of Kazakhstan and Ukraine: the ESSENCE study. J Med Econ. 2018;21(10):1006-15.

6. Li D, Madhoun HM, Roberts WN Jr, Jarjour W. Determining risk factors that increase hospitalizations in patients with systemic lupus erythematosus. Lupus. 2018;27(8):1321-28

7. Centers for Diseases Control and Prevention. Lupus studies. 2017. Available at: https://www.cdc.gov/lupus/funded/lupus-studies.htm. Accessed January 27, 2020.

8. Reilly MC, Zbrozek AS, Dukes EM. The validity and reproducibility of a work productivity and activity impairment instrument. PharmacoEconomics. 1993;4(5):353-65

9. Graham J, Hofer S. Modeling Longitudinal and Multiplegroup Data: Practical Issues, Applied Approaches, and Specific Examples. Mahwah, NJ: Lawrence Erlbaum Associates; 2000.

10. Chakravarty EF, Bush TM, Manzi S, Clarke AE, Ward MM. Prevalence of adult systemic lupus erythematosus in California and Pennsylvania in 2000: estimates obtained using hospitalization data. Arthritis Rheum. 2007;56(6):2092-94.

11. Elixhauser A, Steiner C. Readmissions to U.S. hospitals by diagnosis, 2010. Available at: https://www.hcup-us.ahrq.gov/reports/statbriefs/sb153. pdf. Accessed January 27, 2020.

12. Campbell R, Jr., Cooper GS, Gilkeson GS. The impact of systemic lupus erythematosus on employment. J Rheumatol. 2009;36(11):2470-75.

13. Yelin E, Trupin L, Katz P, et al. Work dynamics among persons with systemic lupus erythematosus. Arthritis Rheum. 2007;57(1):56-63.

14. Bertoli AM, Fernandez M, Alarcon GS, Vila LM, Reveille JD. Systemic lupus erythematosus in a multiethnic U.S. cohort LUMINA (XLI): factors predictive of self-reported work disability. Ann Rheum Dis. 2007;66(1):12-17.

15. Panopalis P, Yazdany J, Gillis JZ, et al. Health care costs and costs associated with changes in work productivity among persons with systemic lupus erythematosus. Arthritis Rheum. 2008;59(12):1788-95.

16. Baker K, Pope J, Fortin P, et al. Work disability in systemic lupus erythematosus is prevalent and associated with socio-demographic and disease related factors. Lupus. 2009;18(14):1281-88.

17. Scofield L, Reinlib L, Alarcon GS, Cooper GS. Employment and disability issues in systemic lupus erythematosus: a review. Arthritis Rheum. 2008;59(10): 1475-79.

18. Rees F, Doherty M, Grainge MJ, Lanyon P, Zhang W. The worldwide incidence and prevalence of systemic lupus erythematosus: a systematic review of epidemiological studies. Rheumatology. 2017;56(11):1945-61.

19. Lee J, Peschken CA, Muangchan C, et al. The frequency of and associations with hospitalization secondary to lupus flares from the 1000 Faces of Lupus Canadian cohort. Lupus. 2013;22(13):1341-48. 
20. Montes RA, Mocarzel LO, Lanzieri PG, Lopes LM, Carvalho A, Almeida JR. Smoking and its association with morbidity in systemic lupus erythematosus evaluated by the Systemic Lupus International Collaborating Clinics/ American College of Rheumatology Damage Index: preliminary data and systematic review. Arthritis Rheum. 2016;68(2):441-48.
21. Kan HJ, Song X, Johnson BH, Bechtel B, O'Sullivan D, Molta CT. Healthcare utilization and costs of systemic lupus erythematosus in Medicaid. BioMed Res Int. 2013;2013:808391. 


\begin{tabular}{|c|c|}
\hline APPENDIX & $\begin{array}{l}\text { Precoded Medications List Provided } \\
\text { to Respondents }\end{array}$ \\
\hline Drug Class & Representative Generic (Brand) Medications \\
\hline Steroid & $\begin{array}{l}\text { Cortisone (Cortone) } \\
\text { Dexamethasone (Decadron) } \\
\text { Hydrocortisone (Cortef) } \\
\text { Prednisone (Deltasone) } \\
\text { Prednisolone (Prelone) } \\
\text { Methylprednisolone (Medrol) } \\
\text { Clobetasol } \\
\text { Cortisone shot } \\
\text { Fluticasone } \\
\text { Triamcinolone cream/ointment (Kenalog) }\end{array}$ \\
\hline Biologic & $\begin{array}{l}\text { Abatacept (Orencia) } \\
\text { Anakinra (Kineret) } \\
\text { Belimumab (Benlysta) } \\
\text { Rituximab (Rituxan and MabThera) } \\
\text { Tocilizumab (Actemra) } \\
\text { Ustekinumab (Stelara) } \\
\text { TNF inhibitors: } \\
\text { Certolizumab pegol (Cimzia) } \\
\text { Adalimumab (Humira) } \\
\text { Etanercept (Enbrel) } \\
\text { Golimumab (Simponi) } \\
\text { Infliximab (Remicade) }\end{array}$ \\
\hline DMARD & $\begin{array}{l}\text { Hydroxychloroquine (Plaquenil) } \\
\text { Leflunomide (Arava) } \\
\text { Methotrexate (Rheumatrex, Trexall, Otrexup, Rasuvo) } \\
\text { Tofacitinib citrate (Xeljanz) } \\
\text { Azathioprine (Azasan, Imuran) } \\
\text { Cyclosporine (Neoral, Sandimmune, Gengraf) } \\
\text { Sulfasalazine (Azulfidine) }\end{array}$ \\
\hline GABA analog & $\begin{array}{l}\text { Gabapentin (Neurontin) } \\
\text { Pregabalin (Lyrica) }\end{array}$ \\
\hline Immunosuppressant & $\begin{array}{l}\text { Mycophenolate mofetil (CellCept) } \\
\text { Mycophenolate sodium (Myfortic) } \\
\text { Tacrolimus (Prograf, Advagraf, Protopic) } \\
\text { Rapamune (Rapamycin) } \\
\text { Pimecrolimus (Elidel) }\end{array}$ \\
\hline Hormone & $\begin{array}{l}\text { Corticotropin injection (Acthar Gel) } \\
\text { Dehydroepiandrosterone }\end{array}$ \\
\hline Other & $\begin{array}{l}\text { Anti-malarials: } \\
\text { Chloroquine (Aralen) } \\
\text { Quinacrine/mepacrine (Atabrine) } \\
\text { Alkylating agent and immunosuppressant: } \\
\text { Cyclophosphamide (Cytoxan) } \\
\text { Antibodies: } \\
\text { Intravenous immunoglobulin } \\
\text { Antibiotics: } \\
\text { Minocycline (Minocin) } \\
\text { Dapsone }\end{array}$ \\
\hline
\end{tabular}

